\title{
Germinación De Semillas De Asclepias Subulata En Condiciones De Casa Sombra Utilizando Ácido Giberélico
}

\author{
Jesús López-Elías, Dr. \\ José Jiménez-León, Dr. \\ Marco A. Huez-López, Dr. \\ Jesús A. Dávila-Loreto \\ Efraín Ávila-Machado \\ Universidad de Sonora, Departamento de Agricultura y Ganadería, \\ Hermosillo Sonora, México. \\ Ramón E. Robles-Zepeda, Dr. \\ Universidad de Sonora, Departamento de Ciencias Químico Biológicas, \\ Hermosillo Sonora, México.
}

\section{Abstract}

The objective of this work was to evaluate the use of gibberellic acid $\left(\mathrm{GA}_{3}\right)$ in the seed germination of Asclepias subulata Decne., plant of the wild flora of the Sonoran Desert of interest for reforestation, industry and medicinal use. Since there is no information about their domestication, it is of fundamental importance to carry out germination tests to ensure their propagation, given the low germination percentage that this species presents. Seeds of A. subulata were collected in the Sonoran Desert, within the area known as Cerro del Bachoco. Six concentrations of gibberellic acid $\left(\mathrm{GA}_{3}\right)$ $(0,125,250,500,1000$ and $2000 \mathrm{ppm})$ were tested at two soak times (24 and 48 hours) and one control without any treatment. Germination power, germination time at 25,50 and $75 \%$, and the germination rate index were evaluated. Germination was considered when the seed emits the hypocotyl outside the substrate used. Chemical scarification with $\mathrm{GA}_{3}$ has a positive influence on seed germination of $A$. subulata. The highest percentage of germination was obtained with $\mathrm{GA}_{3}$ in concentrations of 250 to $2000 \mathrm{ppm}$, at 24 and 48 hours of soaking. Germination time was affected by the concentration of $\mathrm{GA}_{3}$, being favored at a concentration of 1000 and 2000 ppm at 24 and 48 hours of soaking; while in other treatments $75 \%$ of germination was not reached. Chemical scarification with $\mathrm{GA}_{3}$ favored the germination rate index. 
Keywords: Rush milkweed, desert milkweed, stratification, dormancy, seeds

\section{Resumen}

El presente trabajo tuvo como objetivo evaluar el uso de ácido giberélico $\left(\mathrm{GA}_{3}\right)$ en la germinación de semillas de Asclepias subulata Decne., planta de la flora silvestre del Desierto Sonorense de interés para reforestación, industria y uso medicinal. Al no existir información al respecto de su domesticación, es de fundamental importancia realizar pruebas de germinación que permitan asegurar su propagación dado el bajo porcentaje de germinación que presenta esta especie. Semillas de A. subulata fueron colectadas en el Desierto Sonorense, en el área conocida como Cerro del Bachoco. Se probaron seis concentraciones de ácido giberélico $\left(\mathrm{GA}_{3}\right)(0,125$, $250,500,1000$ y $2000 \mathrm{ppm}$ ) a dos tiempos de remojo (24 y 48 horas) y un testigo sin ningún tratamiento. Se determinó el poder germinativo, tiempo de germinación al 25, 50 y $75 \%$ y el índice de velocidad de germinación. La germinación se consideró cuando la semilla emite el hipocotilo sobre el sustrato utilizado. La escarificación química con $\mathrm{GA}_{3}$ ejerce una influencia positiva sobre la germinación de semillas de Asclepias subulata. El mayor porcentaje de germinación se obtuvo con $\mathrm{GA}_{3}$ en concentración de 250 a 2000 ppm, a 24 y 48 horas de remojo. El tiempo de germinación se vio afectado por la concentración de $\mathrm{GA}_{3}$, viéndose favorecido a una concentración de 1000 y 2000 ppm a 24 y 48 horas de remojo; en tanto que en los demás tratamientos no se alcanzó el $75 \%$ de germinación. La escarificación química con $\mathrm{GA}_{3}$ favoreció el índice de velocidad de germinación.

Palabras clave: Algodoncillo, estratificación, jumete, latencia, semillas

\section{Introducción}

Las semillas son el principal mecanismo de reproducción de especies vegetales. Una vez finalizado su desarrollo, estas permanecen en estado de reposo hasta que se dan las condiciones favorables para su germinación. Este estado puede venir determinado por la existencia de condiciones ambientales desfavorables (quiescencia) o por la existencia de factores que actúan desde la propia semilla no permitiendo su germinación (latencia) (Pita y Pérez, 1998).

Un gran número de semillas presentan latencia, razón por la cual no germinan aun cuando sean viables y se expongan a condiciones favorables (Robles, 1990). Los principales factores que inhiben la germinación son físico-mecánicos o físico-químicos, interviniendo en los primeros los recubrimientos de la semilla que influyen en la entrada de agua y oxígeno al 
actuar como barrera mecánica; mientras que en segundo intervienen ácidos o compuestos inhibidores de la germinación (Bidwell, 1990).

Algunos autores (Maguirre, 1976; Jiménez, 1984; Copeland y McDonald, 1985; ISTA, 1985) han descrito tratamientos para vencer la latencia y estimular la germinación en semillas: 1. La escarificación mecánica. Usada en semillas duras o impermeables. Consiste en refregar, dañar o frotar las semillas con superficies abrasivas, como lija, piedra, carbonato de silicio o pueden golpear con un martillo, con la finalidad de facilitar la entrada de agua y el intercambio gaseoso; 2. La escarificación química. Usada para el tratamiento con semillas duras. Generalmente se usa ácido sulfúrico. Las semillas se remojan en una solución concentrada por periodos de tiempo, que varían para cada especie. Existe también la escarificación con agua caliente o a temperatura ambiente, que es una de las técnicas más ampliamente usadas y consiste en sumergir la semilla en agua durante cierto tiempo, para acelerar el proceso de inhibición o mejorar las características de la cubierta. Asimismo, el tratamiento con peróxido de hidrógeno $\left(\mathrm{H}_{2} \mathrm{O}_{2}\right)$ favorece la germinación estimulando tanto la capacidad como la energía germinativa.

Las hormonas vegetales tienen un relevante papel en la germinación de las semillas; sin embargo, aún se desconocen la mayoría de los mecanismos implicados en su actuación. Desde el punto de vista de la germinación, las hormonas vegetales se pueden dividir en dos grupos: a) Promotoras de la germinación, y b) Inhibidoras de la germinación. En el primer grupo destacan las giberelinas, desempeñando un papel clave en los mecanismos fisiológicos relacionados con la germinación (Pita y Pérez, 1998; Kucera et al., 2015).

Las especies forestales suelen presentar una gran variabilidad interespecífica, que es mayor en especies de amplia distribución, y que suele corresponder con adaptaciones a condiciones ecológicas ligadas a las zonas de distribución. Estas variaciones afectan el crecimiento, desarrollo, producción de fruto, y a la germinación de la semilla (Herrero et al., 2001).

Asclepias subulata Decne., llamada comúnmente inmortal, jumete, candelilla bronca, talayote, mata candelilla, hierbajo lechoso, najca'azjc, es una planta herbácea perenne, se localiza en los estados de Baja California, Baja California Sur, Sonora y Sinaloa, a una latitud de 5 a 440 m.s.n.m. Su hábitat son las dunas costeras, bosque tropical caducifolio, vegetación riparia, matorral xerófito y matorral subtropical (Fernández et al., 2008; Oberhauser et al., 2009; Southwest Desert Flora, 2015). Pertenece a la familia Asclepiadaceae, posee tallos fotosintéticos (Borders y Lee-Mäder, 2014), alcanza una altura de $1.2 \mathrm{~m}$ y un ancho de $0.6 \mathrm{~m}$ (Sunnylands, 2013), su floración es esporádica a lo largo del año, en respuesta a la lluvia 
estacional, siendo una de las pocas Asclepias siempre verdes (Borders y LeeMäder, 2014), flores de color crema-amarillo (Sunnylands, 2013).

A. subulata está siendo usada como especie prioritaria para la restauración del hábitat de la mariposa monarca (Oberhauser et al., 2009; Borders y Lee-Mäder, 2014), paisajismo (Bradley, 1998), producción de látex (Hall y Long, 1921; Beckett y Stitt, 1935), obtención de compuestos fenólicos (Agrawal et al., 2009) y uso medicinal (Fernández et al., 2008; Borders y Lee-Mäder, 2014; Rascón et al., 2015). Sin embargo, existe escasa información al respecto su propagación, encontrándose trabajos en los cuales se ha observado que dicha especie presenta baja germinación (Hall y Long, 1921; Wyatt et al., 1996).

Los requerimientos para la germinación de la mayoría de las Asclepias no han sido descritos en la literatura. Las semillas de Asclepias dispersadas en verano o en otoño no germinan, dando inicio la germinación hasta finales del invierno o la primavera cuando las semillas han madurado y la temperatura aumenta coincidiendo con la requerida para la germinación (Borders y Lee-Mäder, 2014).

Borders y Lee-Mäder (2014) mencionan que Asclepias deberá sembrarse en otoño de manera que las temperaturas bajas y la humedad proveniente de las lluvias durante el invierno estimulen la germinación en primavera; pudiendo sembrarse también en primavera, previa estratificación de la semilla a bajas temperaturas $\left(4-5^{\circ} \mathrm{C}\right)$ y manteniendo la humedad en el suelo durante el período de germinación. Posterior a la estratificación, que puede ser por un período de 7 días a 11 meses, la temperatura recomendada para la germinación deberá estar entre los $18-27^{\circ} \mathrm{C}$ (Luna y Dumroese, 2013).

En semillas de Asclepias también puede hacerse uso de la escarificación química. El remojo en nitrato de potasio incrementa significativamente la germinación, aunque en menor grado que la estratificación en frío. En tanto que el remojo con acetona y etanol no afecta significativamente la germinación, el remojo en ácido sulfúrico causa una reducción en la misma. Por otra parte, el tratamiento con reguladores de crecimiento, como la Kinetina y el ácido giberélico, presentan un efecto positivo en la germinación (Borders y Lee-Mäder, 2014).

El ácido giberélico induce la síntesis de $a$-amilasa, enzima que participa en la desintegración de las reservas de almidón durante la germinación de la semilla, por lo cual su uso como promotor o inductor de la germinación en diversas plantas (Bewley y Black, 1994; Baskin y Baskin, 2014).

El objetivo del trabajo fue evaluar el uso de ácido giberélico $\left(\mathrm{GA}_{3}\right)$ en la germinación de Asclepias subulata, bajo condiciones de casa sombra. 


\section{Material y Métodos}

Las semillas de Asclepias subulata Decne. fueron colectadas en el Desierto Sonorense, en el área conocida como Cerro del Bachoco, a los $29^{\circ} 08^{\prime} 43^{\prime \prime} \mathrm{N}$ y $110^{\circ} 57^{\prime} 10^{\prime \prime} \mathrm{O}$. La temperatura media anual es de $25.0^{\circ} \mathrm{C}$ y la precipitación total de $246.4 \mathrm{~mm}$, clima $\mathrm{BW}\left(\mathrm{h}^{\prime}\right) \mathrm{hw}\left(\mathrm{x}^{\prime}\right)\left(\mathrm{e}^{\prime}\right)$ el cual corresponde a la categoría de muy árido, extremoso y cálido (García, 1988).

La colecta se realizó durante el otoño de 2015, a partir de vainas en estado dehiscente; de las cuales se extrajeron semillas maduras, sin presencia de daño y se almacenaron en bolsas de papel bajo condiciones normales de laboratorio.

Para la siembra se usaron charolas de unicel de 200 cavidades y como sustrato una combinación de peat moss y perlita en proporción 1:1. Se colocó una semilla por cavidad, a menos de un centímetro de profundidad, cubriendo con una capa del mismo sustrato. Las charolas se estibaron y envolvieron con plástico para acelerar la germinación. Una vez iniciada la germinación, las charolas se pasaron a una casa sombra cubierta con una malla sombreadero del $30 \%$. En este trabajo, la germinación se consideró cuando la semilla emite el hipocotilo sobre el sustrato utilizado.

La siembra se llevó a cabo el 5 de abril de 2016. Todos los días se revisaba la emergencia hasta la estabilización de la germinación que duró 20 días. Se utilizaron 6 concentraciones de ácido giberélico $\left(\mathrm{GA}_{3}\right)$ de 0,125 , 250,500, 1000 y 2000 ppm a dos períodos de remojo (24 y 48 horas) y un testigo sin ningún tratamiento (Cuadro 1)

Cuadro 1. Tratamientos evaluados en la germinación de semillas de Asclepias subulata.

\begin{tabular}{cc}
\hline TRATAMIENTO & Descripción \\
\hline T & Testigo sin ningún tratamiento \\
GA2000+ & $\mathrm{GA}_{3} 2000 \mathrm{ppm}+$ remojo $48 \mathrm{~h}$ \\
GA1000+ & $\mathrm{GA}_{3} 1000 \mathrm{ppm}+$ remojo $48 \mathrm{~h}$ \\
GA500+ & $\mathrm{GA}_{3} 500 \mathrm{ppm}+$ remojo $48 \mathrm{~h}$ \\
GA250+ & $\mathrm{GA}_{3} 250 \mathrm{ppm}+$ remojo $48 \mathrm{~h}$ \\
GA125+ & $\mathrm{GA}_{3} 125 \mathrm{ppm}+$ remojo $48 \mathrm{~h}$ \\
R+ & $\mathrm{GA}_{3} 0 \mathrm{ppm}+$ remojo $48 \mathrm{~h}$ \\
GA2000- & $\mathrm{GA}_{3} 2000 \mathrm{ppm}+$ remojo $24 \mathrm{~h}$ \\
GA1000- & $\mathrm{GA}_{3} 1000 \mathrm{ppm}+$ remojo $24 \mathrm{~h}$ \\
GA500- & $\mathrm{GA}_{3} 500 \mathrm{ppm}+$ remojo $24 \mathrm{~h}$ \\
GA250- & $\mathrm{GA}_{3} 250 \mathrm{ppm}+$ remojo $24 \mathrm{~h}$ \\
GA125- & $\mathrm{GA}_{3} 125 \mathrm{ppm}+$ remojo $24 \mathrm{~h}$ \\
R- & $\mathrm{GA}_{3} 0 \mathrm{ppm}+$ remojo 24 h \\
\hline
\end{tabular}

El $\mathrm{GA}_{3}$ se obtuvo del producto comercial ProGibb®, que contiene $40 \%$ de ácido giberélico como ingrediente activo. Cada una de las concentraciones se obtuvo a partir de una solución madre, para la cual se disolvieron $5 \mathrm{~g}$ del producto en $20 \mathrm{~mL}$ de alcohol, para posteriormente levar a $1 \mathrm{~L}$ aforando con agua destilada, con lo que se obtiene una concentración 
inicial de $2000 \mathrm{ppm}$. De ahí se tomaron 100, 50, 25, 12.5 y $6.25 \mathrm{~mL}$ y se aforaron a $100 \mathrm{~mL}$, con los que se obtuvo los tratamientos de 2000, 1000, 500,250 y 125 ppm, respectivamente.

Con el número de semillas germinadas en cada tratamiento, se calcularon varios parámetros de germinación y estos fueron:

\section{Poder germinativo}

Es el porcentaje de semillas germinadas al finalizar el ensayo y se calcula mediante la siguiente fórmula:

Dónde:

$$
\mathrm{E}=\frac{\mathrm{n}}{\mathrm{N}} 100
$$

$\mathrm{E}=$ Emergencia, en porcentaje.

$\mathrm{n}=$ Número de semillas germinadas.

$\mathrm{N}=$ Número total de semillas sembradas.

Cuanto mayor es el valor de E obtenido, mayor es la emergencia y consecuentemente mayor vigor.

\section{Tiempo de germinación}

Tiempo necesario para conseguir un porcentaje de germinación determinado. En este trabajo se evaluó el tiempo necesario para alcanzar el $25\left(\mathrm{~T}_{25}\right), 50\left(\mathrm{~T}_{50}\right)$ y $75 \%\left(\mathrm{~T}_{75}\right)$ de la capacidad de germinación.

\section{Índice de velocidad de germinación}

Representa la velocidad en número de semillas germinadas por día (Maguire, 1962) y se calcula mediante la siguiente fórmula:

Donde:

$$
I V G=\Sigma \frac{G_{i}}{N_{i}}
$$

IVG = Índice de velocidad de germinación, en semillas por día.

$\mathrm{G}_{\mathrm{i}}=$ Número de semillas germinadas al día i.

$\mathrm{N}_{\mathrm{i}}=$ Tiempo transcurrido desde la siembra hasta el día i, en días.

El experimento se estableció en un diseño experimental de bloques completos a azar, con cuatro repeticiones y 10 unidades experimentales por tratamiento. Para el análisis de los datos se utilizó el programa SAS 8.2 (SAS Institute Inc., 2001); se realizó el análisis de varianza de los datos, obteniéndose también la prueba de rango múltiple de Duncan con nivel de significancia del $5 \%$. 


\section{Resultados y Discusión \\ Poder germinativo}

Los porcentajes de germinación variaron entre tratamientos, promediando un $77.0 \%$. Las semillas de Asclepias subulata presentaron el mayor porcentaje de germinación a concentraciones de $\mathrm{GA}_{3}$ de 250, 500, 1000 y 2000 ppm, en ambos tratamientos de remojo (24 y 48 horas), correspondiendo el más bajo al testigo con un 40\% (Cuadro 2). En la figura 1 se presenta la dinámica de germinación de $A$. subulata en valores porcentuales acumulados.

El bajo porcentaje de germinación en el testigo coincide con los estudios realizados por Godínez-Alvarez y Flores-Martínez (1999), al igual que Pece et al. (2010a), quienes encontraron que la imbibición y la escarificación química dio buenos resultados en la germinación, siendo mayor el efecto con la escarificación; coincidiendo al igual con los resultados obtenidos por Pece et al. (2010b), evaluando el efecto de la escarificación química en la germinación.

Los resultados obtenidos coinciden también con aquellos de Araiza et al. (2011), quienes en un estudio con semillas de chiltepín tratadas previamente con $\mathrm{GA}_{3}$, encontraron que su uso estimuló un mayor porcentaje de germinación; lo que también fue observado en estudios realizados por Abdel-Hamid y Mohamed (2014), evaluando $\mathrm{GA}_{3}$ en semillas de cebada.

Cuadro 2. Emergencia, tiempo de germinación e índice de velocidad de germinación de semillas de Asclepias subulata en condiciones de casa sombra utilizando ácido giberélico.

\begin{tabular}{cccccc}
\hline TRATAMIENTO & $\begin{array}{c}\mathrm{E} \\
(\%)\end{array}$ & $\begin{array}{c}\mathrm{T}_{25} \\
(\text { días})\end{array}$ & $\begin{array}{c}\mathrm{T}_{50} \\
(\text { días })\end{array}$ & $\begin{array}{c}\mathrm{T}_{75} \\
(\text { días })\end{array}$ & $\begin{array}{c}\text { IVG } \\
\text { (semillas día } \\
1 \text { ) }\end{array}$ \\
\hline $\mathrm{T}$ & $40.0 \mathrm{c}$ & $15.0 \mathrm{a}$ & $*$ & $\mathrm{x}$ & $0.337 \mathrm{c}$ \\
GA2000+ & $90.0 \mathrm{a}$ & $6.0 \mathrm{~b}$ & $8.7 \mathrm{bc}$ & $13.0 \mathrm{a}$ & $1.273 \mathrm{ab}$ \\
GA1000+ & $80.0 \mathrm{a}$ & $5.7 \mathrm{~b}$ & $6.3 \mathrm{c}$ & $14.0 \mathrm{a}$ & $1.280 \mathrm{ab}$ \\
GA500+ & $86.7 \mathrm{a}$ & $5.0 \mathrm{~b}$ & $7.3 \mathrm{c}$ & $*$ & $1.330 \mathrm{ab}$ \\
GA250+ & $83.3 \mathrm{a}$ & $5.3 \mathrm{~b}$ & $6.3 \mathrm{c}$ & $*$ & $1.440 \mathrm{a}$ \\
GA125+ & $73.3 \mathrm{ab}$ & $4.7 \mathrm{~b}$ & $11.7 \mathrm{abc}$ & $*$ & $1.407 \mathrm{a}$ \\
R+ & $46.7 \mathrm{bc}$ & $5.5 \mathrm{~b}$ & $*$ & $\mathrm{x}$ & $0.673 \mathrm{bc}$ \\
GA2000- & $80.0 \mathrm{a}$ & $6.7 \mathrm{~b}$ & $7.7 \mathrm{c}$ & $13.5 \mathrm{a}$ & $1.090 \mathrm{ab}$ \\
GA1000- & $86.7 \mathrm{a}$ & $6.7 \mathrm{~b}$ & $9.7 \mathrm{abc}$ & $14.7 \mathrm{a}$ & $1.077 \mathrm{ab}$ \\
GA500- & $86.7 \mathrm{a}$ & $6.0 \mathrm{~b}$ & $9.7 \mathrm{abc}$ & $*$ & $1.120 \mathrm{ab}$ \\
GA250- & $80.0 \mathrm{a}$ & $8.7 \mathrm{~b}$ & $14.3 \mathrm{ab}$ & $*$ & $0.860 \mathrm{abc}$ \\
GA125- & $63.3 \mathrm{abc}$ & $8.7 \mathrm{~b}$ & $15.3 \mathrm{a}$ & $*$ & $0.807 \mathrm{abc}$ \\
R- & $66.7 \mathrm{abc}$ & $5.7 \mathrm{~b}$ & $*$ & $*$ & $1.080 \mathrm{ab}$ \\
\hline
\end{tabular}




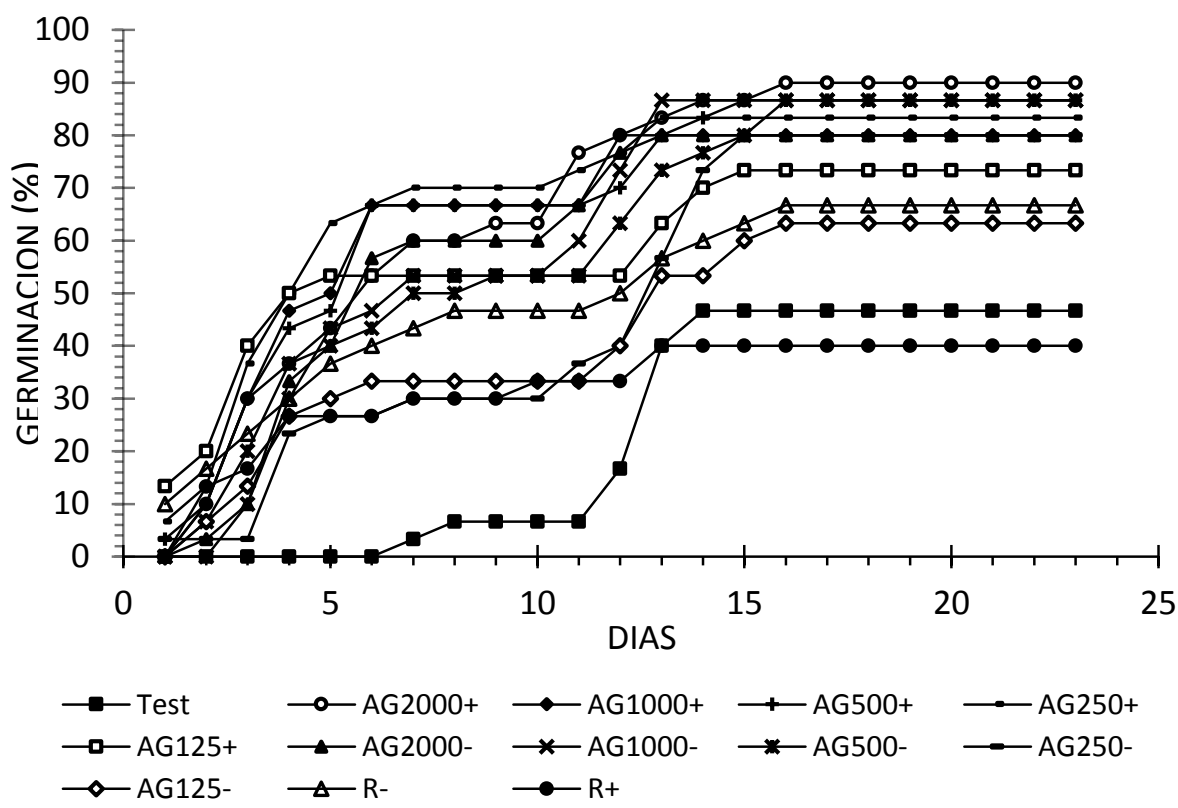

Figura 1. Dinámica de germinación de semillas de Asclepias subulata en valores porcentuales acumulados.

\section{Tiempo de germinación}

Los resultados obtenidos con respecto al número de días requeridos para alcanzar el 25, 50 y $75 \%$ de semillas germinadas se presentan en el cuadro 1. Como puede observarse, para un $\mathrm{T}_{25}$ el menor tiempo de germinación se obtuvo en aquellos tratamientos con remojo de 48 horas, promediando 5.2 días, sin diferencias significativas entre tratamientos; en tanto que el testigo presentó el mayor tiempo de germinación, requiriendo de 15 días para alcanzar el 25\% de semillas germinadas.

Para alcanzar un $\mathrm{T}_{50}$, el menor tiempo de germinación se obtuvo en aquellos tratamientos con remojo de 48 horas, con un promedio de 8.1 días, siendo este menor en los tratamientos con $\mathrm{GA}_{3}$ a 250, 500 y 1000 ppm; en tanto que en el testigo, al igual que en los tratamientos solo con remojo a 24 y 48 horas, no en todas las repeticiones se obtuvo el $50 \%$ de semillas germinadas.

En cuanto al tiempo requerido para alcanzar un $\mathrm{T}_{75}$, el menor tiempo de germinación se obtuvo en aquellos tratamientos con remojo de 48 horas, promediando 13.5 días; en tanto que en el testigo, al igual que en el tratamiento solo con remojo a 48 horas no alcanzaron el $75 \%$ de semillas germinadas. En los tratamientos con $\mathrm{GA}_{3}$ a 125,250 y $500 \mathrm{ppm}$ a 24 y 48 horas de remojo, al igual que en el tratamiento con solo 24 horas de remojo, no en todas las repeticiones se obtuvo el $75 \%$ de semillas germinadas. 
Si bien todos los tratamientos tuvieron el $25 \%$ de germinación, no fue así para el 50 y $75 \%$ en donde no todos los tratamientos alcanzaron a germinar. Resultados que coinciden con Pece et al. (2010a), evaluando la imbibición y escarificación química en semillas de Pterogyne nitens Tul.

\section{Índice de velocidad de germinación}

En cuanto al índice de velocidad de germinación (IVG), como puede observarse en el cuadro 1, el índice más alto se obtuvo en aquellos tratamientos con remojo de 48 horas, promediando un IVG de 1.234, siendo este mayor en los tratamientos con $\mathrm{GA}_{3}$ a 125 y 250 ppm, con un promedio de 1.424 semillas día ${ }^{-1}$, sin diferencias significativas entre tratamientos; en tanto que el testigo, con 0.337 semillas día ${ }^{-1}$, presentó el menor IVG.

Los resultados coinciden con Pece et al. (2010a) quienes, evaluando la germinación de Pterogyne nitens, encontraron que los tratamientos usados para la escarificación química influyeron significativamente en el IVG; sin diferencias entre ellos, aunque si con respecto al testigo que presentó el menor índice.

El bajo IVG en el testigo coincide también con los trabajos realizados por Pece et al. (2010b), quienes encontraron que la escarificación química favoreció la velocidad de germinación en Tipuana tipu.

\section{Conclusiones}

La escarificación química con $\mathrm{GA}_{3}$ ejerce una influencia positiva sobre la germinación de semillas de Asclepias subulata. El mayor porcentaje de germinación se obtuvo con $\mathrm{GA}_{3}$ en concentración de 250 a 2000 ppm, a 24 y 48 horas de remojo. El tiempo de germinación se vio afectado por la concentración de $\mathrm{GA}_{3}$, viéndose favorecido a una concentración de $1000 \mathrm{y}$ 2000 ppm a 24 y 48 horas de remojo; en tanto que a otras concentraciones no todas las repeticiones alcanzaron el 75\% de germinación. La escarificación química con $\mathrm{GA}_{3}$ favoreció el índice de velocidad de germinación.

\section{References:}

1. Abdel-Hamid, A. M. E., \& Mohamed, H. I. (2014). The effect of the exogenous gibberellic acid on two salt stressed barley cultivars. European Scientific Journal, 10, 228-245.

2. Araiza, N., Araiza, E., \& Martínez, J. G. (2011). Evaluación de la germinación y crecimiento de plántula de chiltepín (Capsicum annuum L variedad glabriusculum) en invernadero. Rev. Colomb. Biotecnol, 13, 170-175.

3. Baskin, C. C., \& Baskin, J. M. (2014). Seeds: Ecology, biogeography, and, evolution of dormancy and germination. Elsevier. 
4. Beckett, R. E., \& Stitt, R. S. (1935). The desert milkweed (Asclepias subulata) as a possible source of rubber. United States Department of Agriculture. Technical Bulletin No.472. U.S.A.

5. Bewley, J. D., \& Black, M. (1994). Seeds: Physiology of development and germination. Springer Science \& Business Media.

6. Bidwell, R. G. S. (1990). Fisiología vegetal. AGT Editor, S.A. México.

7. Borders, B., \& Lee-Mäder, E. (2014). Milkweeds: A conservation practitioner's guide. The Xerces Society for Invertebrate Conservation. U.S.A.

8. Bradley, L. (1998). Plants for poolside landscapes. The University of Arizona. Publication AZ1058 8/98. U.S.A.

9. Copeland, L. O., \& McDonald, M. B. (2001). Principles of seed science and technology. Springer Science and Business Media. U.S.A.

10. Enríquez-Peña, E. G., Suzán-Azpiri, H., \& Malda-Barrera, G. (2004). Viabilidad y germinación de semillas de Taxodium mucronatum (Ten.) en el estado de Querétaro, México. Agrociencia, 38, 375-381.

11. Fernández, A. M., Juárez, V., \& Cortés, L. (2008). Uso de las especies del género Asclepias L. (Apocynaceae, Asclepiadoideae), información del herbario nacional de México, MEXU. Polibotánica, $25,155-171$.

12. García, E. (1988). Modificaciones al sistema de clasificación climática de Köppen. Offset Larios. México.

13. Godínez-Alvarez, H., \& Flores-Martínez, A. (1999). Germinación de semillas de 32 especies de la Costa de Guerrero: su utilidad para la restauración ecológica. Polibotánica, 11, 1-19.

14. Hall, H. M., \& Long, F. L. (1921). Rubber-Content of North American plants. Carnegie Institution of Washington. Publication No.313. U.S.A.

15. Herrero, N., Benito, L. F., Carrasco, I., \& Peñuelas, J. L. (2001). Nuevos pretratamientos para la germinación de Fagus sylvatica L. Actas del III Congreso Forestal Español. Granada, España, 3, 647652.

16. ISTA. (1985). Rules for seed testing technology. International Seed Testing Association. Seed Science and Technology, 13, 299,355.

17. Jiménez, M. A. (1984). Análisis de la calidad de la semilla de especies forrajeras tropicales. Universidad Autónoma de Chapingo. Depto. de Zootecnia. México.

18. Luna, T., \& Dumroese, R. K. (2013). Monarchs (Danaus plexippus) and milkweeds (Asclepias species): The current situation and methods for propagating milkweeds. Native Plants Journal, 14, 5-15. 
19. Maguire, J. D. (1962). Speeds of germination-aid selection and evaluation for seedling emergence and vigor. Crop Sci., 2, 176-177.

20. Maguirre, J. D. (1976). Seed dormancy advances in research and technology of seed. Washington State University. Dept. Agronomy and Soils. U.S.A.

21. Oberhauser, K., Batalden, R., \& Howard, E. (2009). Monitoreo de la mariposa monarca en américa del norte: resumen de iniciativas y protocolos. Comisión para la Cooperación Ambiental. Canadá.

22. Kucera, B., Cohn, M. A., \& Leubner-Metzger, G. (2005). Plant hormone interactions during seed dormancy release and germination. Seed Science Research, 15, 281-307.

23. Pece, M., Gaillard, C., Acosta, M., Bruno, C., \& Saavedra, S. (2010a). Tratamientos pregerminativos para tipa colorada (Pterogyne nitens Tul.). Foresta Veracruzana, 12, 17-25.

24. Pece, M.G., Gaillard, C., Acosta, M., Bruno, C., Saavedra, S., \& Buvenas, O. (2010b). Germinación de Tipuana tipu (Benth.) O. Kuntze (tipa blanca) en condiciones de laboratorio. Revista de Ciencias Forestales - Quebracho, 18, 5-15.

25. Pita, J. M., \& Pérez, F. (1998). Germinación de semillas. Ministerio de Agricultura, Pesca y Alimentación. Núm. 2090 HD. Madrid, España.

26. Rascón, L. A., Jiménez, M., Velázquez, C. A., Garibay, A., Medina, L. A., Gámez, N., \& Robles, R. E. (2015). Antiproliferative and apoptotic activities of extracts of Asclepias subulata. Pharmaceutical Biology, 53, 1741-1751.

27. Rodríguez, I., Adam, G., \& Durán, J. M. (2008). Ensayos de germinación y análisis de viabilidad y vigor de semillas. Revista Agropecuaria, 78, 836-842.

28. Robles, R. (1990). Producción de Granos y Forrajes. Limusa. 5a ed. México.

29. SAS Institute Inc. (2001). The SAS System for Windows Release 8.2. Cary, N. C. USA.

30. Southwest Desert Flora. (2015). Asclepias subulata, Rush Milkweed. Home to the plants of the Sonoran, Chuhuahuan, Mojave and Great Basin Deserts. U.S.A.

31. Sunnylands. (2013). Sunnylands center \& gardens. Gardens guide. The Annenberg Foundation Trust at Sunnylands. U.S.A.

32. Wyatt, R., Ivey, Ch. T., \& Lipow, S. R. (1996). The breeding system of desert milkweed, Asclepias subulata. Torrey Botanical Club, Bulletin No.123. 